Revision - Revisión

\title{
USO DAS PRECAUÇÕES-PADRÃO NA ASSISTÊNCIA DE ENFERMAGEM: UM ESTUDO RETROSPECTIVO
}

\author{
The use of the precautions pattern in the nursing attendance: \\ retrospective study \\ El uso de las precauciones el modelo en la asistencia de enfermería: \\ estudio retrospectivo
}

Daniele Fernandes de Aguiar ${ }^{1}$

Aline Bárbara Garcia Lima²

Rita Batista Santos ${ }^{3}$

\section{RESUMO}

Este estudo tem como objetivos analisar as publicações científicas e técnicas sobre os procedimentos de precauções-padrão em periódicos nacionais e internacionais e destacar aspectos importantes dos artigos no que se refere ao papel da enfermeira na prevenção e controle da infecção hospitalar. Constituíram como amostra da pesquisa as fontes secundárias em forma de livros e periódicos, com a finalidade de nos colocar em contato direto com os artigos levantados sobre o uso das precauções-padrão para as infecções hospitalares na assistência de enfermagem, entre 1999 e 2005. Foram encontrados 10 artigos relacionados ao uso de precauções-padrão: $70 \%$ foram obtidos em Banco de Teses, 20\%, no site Scielo e 10\%, na biblioteca de uma universidade pública do Rio de Janeiro, sendo 40\% publicados no ano de 2003. Os resultados mostraram que, neste período, apesar de poucas publicações, houve uma contínua renovação do tema, tanto a nível nacional, quanto internacional.

Palavras-chave: Enfermagem. Infecção Hospitalar. Precauções Universais.

\begin{abstract}
This study has as objectives, analyze the scientific and technical publications about the standard procedures of precautions in national and international newspapers and to highlight important aspects of articles about the nurse's paper in the prevention and control of hospital infection. It constituted as sample of the research the secondary sources in form of books and newspaper, with the purpose of putting us in direct contact with articles raised about the use of the precautions standard for the hospital infections in the nursing care, between 1999 and 2005. 10 articles related to the use of standard precautions had been found: $70 \%$ had been gotten in Bank of Teses, $20 \%$ in the site Scielo and $10 \%$ in the library of a public university of Rio de Janeiro, being $40 \%$ published in the year of 2003 . The results showed that, in this period, in spite of few publications, there was a continuous renewal of the theme, such as national level, as international.
\end{abstract}

Keywords: Nursing. Cross Infection. Universal Precautions.

\section{Resumen}

Este estudio tiene como objetivos analizar las publicaciones científicas y técnicas, que los procedimientos de las precauciones estándares en periódicos nacionales e internacionales, separar aspectos importantes de artículos acerca del papel de la enfermera en la prevención y controlar la infección en hospital. Ellos constituirán como proba, los orígenes secundarios en la forma de libros y periódicos de la investigación, con el propósito de ponernos en contacto directo con los artículos en el uso de las precauciones estándares para las infecciones del hospital en la atención de enfermería, entre 1999 y 2005 con el uso de las precauciones. 10 artículos se relacionaron con el uso de precauciones habían sido encontrado: $70 \%$ habían sido conseguidos en Banco de Teses, el $20 \%$ en el sitio Scielo y el $10 \%$ en la biblioteca de una universidad pública de Río de Janeiro, siendo $40 \%$ publicado en el año de 2003. Los resultados mostraban que, en este período, a pesar de pocas publicaciones, había una renovación continua del tema, tanto en el nivel nacional, como internacional.
Palabras clave: Enfermería. Infección Hospitalaria. Precauciones Universales.

Enfermeira graduada em agosto de 2006 pela Escola de Enfermagem Anna Nery, da Universidade Federal do Rio de Janeiro. Ex-bolsista de Iniciação Científica da FAPERJ. Mestranda em Enfermagem pela EEAN / UFRJ. E-mail: danifaguiarufrj@yahoo.com.br. ${ }^{2}$ Enfermeira graduada em agosto de 2006 pela Escola de Enfermagem Anna Nery, da Universidade Federal do Rio de Janeiro. Residente em Enfermagem Médico-Cirúrgica pela Marinha do Brasil. E-mail: barbaraglima@yahoo.com.br. ${ }^{3}$ Professora Doutora do Departamento de Enfermagem Médico-Cirúrgica da Escola de Enfermagem Anna Nery, da Universidade Federal do Rio de Janeiro. Orientadora da pesquisa. E-mail: rita@eean.ufri.br ; ritabatistas@hucff.ufri.br 


\section{CONSIDERAÇÕES INICIAIS}

As infecções hospitalares podem ser definidas como as infecções que ocorrem em pacientes durante a hospitalização, com diagnóstico confirmado por exames clínicos e de laboratório. Os microorganismos contagiosos podem se originar de fontes endógenas, como a flora comensal endógena da qual o paciente é portador, ou de fontes exógenas, como aquisição recente através de objetos animados ou inanimados dentro do hospital ${ }^{1}$.

Vários fatores podem influenciar na ocorrência das infecções hospitalares, tais como a fonte de infecção, o agente infeccioso, a via de transmissão, a susceptibilidade do hospedeiro e 0 meio ambiente ${ }^{2}$.

Temos como fontes imediatas de microorganismos infecciosos no hospital as pessoas, ou seja, funcionários do hospital, visitantes e pacientes que tenham doença clínica, portadores assintomáticos. Além desses, estão os fômites, que são objetos inanimados, exceto os alimentos, consistindo em material e equipamentos médicos tais como móveis, cobertores, umidificadores, equipamento de anestesia, medicamentos e soluções. Estas últimas podem ser soluções de uso endovenoso contaminadas por bactérias ou fungos, sangue e seus derivados contaminados por vírus. Incluem-se também os alimentos e água contaminados por microorganismos, como por exemplo Salmonella ou Shiguella. Os animais ou insetos constituem igualmente fontes importantes de contaminação ${ }^{2}$.

Com relação aos agentes infecciosos, há muitas espécies aos quais o paciente fica exposto durante a hospitalização. A probabilidade de infecção resultante dessa exposição depende em parte da espécie de agente patogênico, da sua resistência aos agentes antimicrobianos administrados ao paciente, da sua virulência e número introduzido no paciente. Dependendo do método de transmissão e dos fatores hospedeiros, qualquer microorganismo pode ser patógeno².

Os microorganismos podem ser transmitidos dentro do ambiente hospitalar por uma das quatro vias: contato, ar, veículo comum e vetor ${ }^{2}$. Mais de uma via pode servir às vezes de transmissão do agente patogênico durante um único episódio, e esse mesmo agente pode ser transmitido por diferentes vias em diferentes ocasiões.

Quanto à susceptibilidade do hospedeiro, seus fatores mais significativos consistem na idade, condições de imunização, tipo da doença básica e efeitos do procedimento de diagnóstico e da terapêutica, como por exemplo, uma queda da resistência a infecç̃os, associada à infância, à velhice e também a pacientes com doenças crônicas, os quais podem ser mais suscetíveis a infecções hospitalares que os outros pacientes. Há ainda muitos procedimentos de diagnóstico, como a biópsia, a cateterização e aspiração de fluidos, que tendem a aumentar o risco de infecções no paciente ${ }^{3}$.

A aglomeração que se verifica no meio ambiente hospitalar também favorece a transmissão de microorganismos. As mudanças da temperatura ou da umidade do ar podem igualmente influenciar a ocorrência de infecções ${ }^{3}$. Por isso, os profissionais que fazem parte da equipe hospitalar têm um papel importante no controle da infecção neste ambiente e um objetivo comum, que é o de zelar pela boa saúde do paciente.

Para que esse objetivo seja alcançado, é necessário que os métodos de prevenção sejam adotados antes mesmo da internação do paciente, através da melhoria das condições sanitárias, do aumento dos serviços básicos de saúde e do tratamento da doença em tempo hábil, a fim de evitar as internações desnecessárias, como já foi dito anteriormente. Deve ser dada a devida importância na fiscalização junto às farmácias, como forma de evitar o uso abusivo de antimicrobianos. Os hospitais, além de equipamentos adequados, devem adotar medidas habituais de prevenção e controle de infecções, através das precauções-padrão. Para tanto, faz-se importante também a aquisição, em seus quadros, de profissionais especializados, de modo a assegurar os princípios técnicos e científicos disponíveis ao controle das infecções hospitalares, incluindo-se entre esses a enfermeira.

Tendo em vista a relevância do assunto acerca da qualidade da assistência prestada aos pacientes hospitalizados portadores de infecção hospitalar, tornou-se necessária a exploração desta temática para controlar a disseminação das infecções nosocomiais e promover-thes os devidos cuidados de enfermagem. A obtenção de tais informações através de artigos científicos publicados em periódicos da área de enfermagem constituiu-se a situação-problema.

Pelos motivos descritos, resolvemos:

- destacar aspectos importantes dos artigos no que se refere ao papel da enfermeira na prevenção e controle da infecção hospitalar;

- analisar as publicações científicas e técnicas sobre os procedimentos de precauções-padrão em periódicos nacionais e internacionais.

Acreditamos que o tema em discussão contribuirá para a pesquisa a fim de divulgar maior número de artigos científicos para tornar as informações acessíveis ao público-alvo, ou seja, a toda a equipe de enfermagem, promovendo a atualização do conhecimento e garantindo uma assistência de melhor qualidade prestada por profissionais de saúde aos clientes hospitalizados para prevenir e/ou combater infecções. 


\section{METODOLOGIA}

Esta é uma revisão de literatura a partir de um levantamento nas bases de dados Scielo, na biblioteca de enfermagem de uma universidade pública do Rio de Janeiro, acervo bibliográfico disponível, Minerva (BVS/UFRJ) e Bireme, Banco de Teses da CAPES e sites de bibliotecas universitárias do estado do Rio de Janeiro e no descritor "Infecção Hospitalar".

A pesquisa constou das seguintes fases: a escolha do tema; a elaboração do plano de trabalho, ou seja, utilizamos descritores baseados na revisão crítica da literatura; a identificação, em que os descritores foram utilizados para obtenção das fontes de informações; fichamento, com o detalhamento dos itens listados na fase de leitura do material; análise e interpretação da literatura sobre os papéis desempenhados pela enfermeira no uso de precauções-padrão; e, finalmente, a redação do relatório ${ }^{4}$.

Constituíram como amostra fontes secundárias, conforme descritas por Eco ${ }^{5}$, em forma de livros e artigos de periódicos, com a finalidade de nos colocar em contato direto com os artigos levantados sobre o uso das precauções-padrão para as infecç̃oes hospitalares na assistência de enfermagem e permitir o reforço paralelo na análise de nossa pesquisa, bem como a manipulação destas informações para a fixação da aprendizagem na nossa formação profissional enquanto acadêmicas do sexto período do curso de graduação em Enfermagem.

No início da pesquisa, pretendíamos utilizar somente como bases de dados o Scielo, a biblioteca de enfermagem de uma universidade pública do Rio de Janeiro, acervo bibliográfico disponível, Minerva (BVS/UFRJ) e Bireme, com os quais localizaríamos artigos e textos científicos especializados na área de enfermagem em infecção hospitalar.

Diante das dificuldades no que diz respeito à exigüidade de tempo para a conclusão do estudo, inserimos dados obtidos no Banco de Teses da CAPES e sites de bibliotecas universitárias do estado do Rio de Janeiro.

A leitura do material foi possível a partir de matriz de itens organizados num instrumento de coleta de dados, as quais permitiram a identificação de autor, ano de publicação, título, indicação para profissionais de enfermagem, método, procedimentos e instrumentos.

A organização dos dados deu-se através de classificação em fichas catalográficas em que apresentamos uma visão de conjunto, um apanhado amplo, feito após um primeiro e superficial contato com o material. Lemos apenas o sumário, as orelhas, o prefácio e a introdução.

A partir do universo de 100 textos encontrados em periódicos nacionais e internacionais, enumeramos e sorteamos 10\% $(n=10)$ destes para constituir a amostra a partir da aplicação da tabela de números aleatórios.

Foram estabelecidos como recorte temporal os anos de 1999 a 2005, com o propósito de obter dados mais recentes acerca do tema.

A análise dos resultados foi realizada à luz do referencial teórico do estudo.

\section{APRESENTAÇÃO E ANÁLISE DOS RESULTADOS}

Para melhor compreensão do tema é importante explicitar que se entendem por precauções-padrão os procedimentos que devem ser adotados em estabelecimentos de saúde durante a assistência a qualquer paciente com processo infeccioso e/ou suspeita de contaminaçãa $0^{6}$, objetivando reduzir o risco de transmissão de microorganismos de fontes de infecção, sejam elas conhecidas ou não. São considerados precauções-padrão o uso de equipamentos de proteção individual (máscaras, luvas, avental, óculos protetores e escudo facial), vacinas e, principalmente, a mais antiga e mais eficiente, a lavagem das mãos com água e sabão ${ }^{6,7,8}$.

Os procedimentos invasivos desnecessários devem ser evitados, e os critérios de prescrição de antibióticos devem ser observados com rigor pelos profissionais de enfermagem e da área médica.

A infecção nosocomial existe em todo o mundo, tanto nos países desenvolvidos como nos subdesenvolvidos. Infecções adquiridas em instituições de saúde estão entre as mais importantes causas de morte e aumento da morbidade nos pacientes hospitalizados. Constituem um peso significativo tanto para os pacientes como para a saúde pública ${ }^{3}$.

Nesse estudo, foram selecionados e analisados 10 artigos sobre o uso das precauções-padrão para a prevenção e controle das infecções hospitalares.

Encontramos como temas: o imaginário social e o cuidado de enfermagem para a clientela portadora de doenças infecciosas e parasitárias, o estudo comparativo do diagnóstico da infecção durante e após a internação, a lavagem das mãos para os trabalhadores de enfermagem, a avaliação de exposição ocupacional, o uso de diagramas de controle na vigilância epidemiológica, o controle de infecção hospitalar no norte de Minas Gerais, a prevalência de estafilococos resistentes à meticilina, as infecções do sítio cirúrgico em pacientes submetidos à cirurgia do aparelho digestivo, o controle de egresso cirúrgico e o modelo aplicativo da adesão às precauções-padrão.

Este resultado revela a inserção das enfermeiras em pesquisas relacionadas ao assunto infecção hospitalar, favorecendo a atualização deste tema, já que a busca limitouse a informações recentes.

No Banco de Teses da CAPES encontramos $70 \%$ dos artigos. Vinte por cento (20\%) foram obtidos na base de dados Scielo e 10\%, na biblioteca de enfermagem de uma universidade pública do Rio de Janeiro. Este resultado demonstra maior facilidade de obter informações através de base de dados virtuais.

Baseando-se nas referências das pesquisas, $80 \%$ delas foram elaboradas por enfermeiras, enquanto $20 \%$ foram elaboradas por médicos. Com isso, verifica-se que há maior interesse por parte das enfermeiras em relação ao uso das precauções-padrão com intuito de garantir uma assistência segura e eficaz ao paciente. 
A estrutura ideal para o controle da infecção hospitalar considera o tipo, as necessidades e os recursos de cada instituição. Deve ser assegurada à equipe de controle de infecção autoridade suficiente para gerir um programa de controle eficaz.

A enfermeira, categoria profissional responsável por $80 \%$ das publicações deste estudo, quando atua na assistência hospitalar, pelo menos em nossa óptica, é responsável por participar na comissão de controle de infecção, contribuir para 0 desenvolvimento e o aperfeiçoamento das técnicas de enfermagem nos programas de formação para enfermeiros e supervisionar a implementação de técnicas para a prevenção de infecção em áreas especializadas.

Outras ações recomendáveis devem ser mantidas, por exemplo, a higiene do ambiente de trabalho, de acordo com as políticas do hospital e as práticas de enfermagem; monitorizar as técnicas assépticas, incluindo a lavagem das mãos e a utilização do isolamento; pedir colheita de amostras para cultura, em qualquer doente com sinais de infecção ou doença transmissível, quando o médico não estiver imediatamente disponível; limitar a exposição dos doentes a infecções provenientes das visitas, dos profissionais, de outros doentes ou de equipamentos utilizados para diagnóstico ou tratamento; e manter provisões adequadas e seguras de equipamentos, fármacos e material para os cuidados dos doentes 3,7,9,9,10.

A enfermeira responsável pelo controle de infecção é membro da equipe deste programa e responsável por identificar infecções nosocomiais; investigar o tipo de infecção e 0 microorganismo causal; participar na formação dos profissionais; fazer a vigilância epidemiológica das infecções hospitalares; participar na investigação de surtos; desenvolver políticas de controle de infecções; rever e aprovar políticas para cuidados dos doentes, que sejam relevantes para o controle de infecção; assegurar o cumprimento dos regulamentos locais e nacionais; fazer a ligação com a saúde pública ou outra instituição, quando apropriado; fornecer consultoria especializada a profissionais de saúde ou a outros programas do hospital, em assuntos relacionados com a transmissão de infecções ${ }^{2,11}$.

A ocorrência de fatores associados à deficiência no controle da infecção hospitalar pelos profissionais acarretam uma interação fisiológica entre um hospedeiro humano e um microorganismo e um grau de patologia subseqüente sofrida pelo ser humano. Elas podem ou não ser comunicáveis (contagiosas) $)^{6}$.

Portanto, as infecções hospitalares no cenário de Doenças Infecciosas e Parasitárias (DIP) merecem uma atenção especial para seu controle, através da educação, supervisão e treinamento da equipe de enfermagem, a fim de reduzir a sua incidência e evitar possíveis demoras nas internações. A equipe deve estar capacitada para cuidar destes pacientes com as devidas precauções.
Sobre a origem da pesquisa, $80 \%$ dos artigos analisados eram nacionais e $20 \%$ internacionais. Apesar de a maioria se concentrar em nível nacional, trata-se de um tema em fase de divulgação a nível internacional.

Segundo o local de realização da pesquisa, $40 \%$ das pesquisas foram realizadas no estado de São Paulo; 30\%, em Minas Gerais; $10 \%$, no Rio de Janeiro; $10 \%$, no Rio Grande do Sul; e 10\%, em Goiás.

Tais dados corroboram a afirmação da Agência Nacional de Vigilância Sanitária (ANVISA) ${ }^{3}$ de que a região Sudeste é a que apresenta o maior índice de doenças adquiridas por infecção hospitalar (16,4\%), seguida do Nordeste (13,1\%), Norte $(11,5 \%)$ e do Sul $(9 \%)$.

Com relação ao ano de realização da pesquisa, $40 \%$ delas foram realizadas no ano de 2003; $30 \%$, em $2001 ; 20 \%$, em 2000; e 10\%, em 1999. 0 avanço das técnicas de precauçõespadrão e o aprimoramento de sua utilização requerem constante atualização do tema através das pesquisas, dessa forma favorecendo a redução de infecções nosocomiais.

Verifica-se que há necessidade de maior controle dos hospitais na redução das infecções hospitalares, visto que nem todas as exigências feitas pela ANVISA têm sido atendidas e, com isso, tem chamado a atenção de profissionais de saúde para a realização de pesquisas acerca deste assunto ${ }^{3}$.

Para este controle ser mais bem efetuado, são necessários diversos procedimentos, como: vigilância epidemiológica; o reconhecimento da importância de todos os fluidos corporais, secreções e excreções na transmissão de patógenos nosocomiais; a adoção de precauções adequadas no controle infeccioso de doenças transmitidas pelo ar, perdigotos e por contato; e o desenvolvimento de atividades assistenciais de acordo com as recomendações da Comissão de Controle de Infecção Hospitalar $(\mathrm{CClH})$, trabalhando-se em parceria com esta 6 .

Os profissionais de saúde que prestam assistência direta devem saber quais são as infecções que afetam mais freqüentemente seus pacientes. Isso possibilita que eles fiquem mais alerta aos primeiros sinais e/ou sintomas de infecção e intervenham imediatamente, se necessário.

Outras razões importantes para a prevenção e controle das infecções hospitalares são os processos judiciais aos quais estão expostos os hospitais e, conseqüentemente, os profissionais de saúde. Em matéria penal, a ocorrência de infecção hospitalar enquadra-se, na maioria das vezes, nas lesões corporais, que podem ser de natureza leve, grave ou até mesmo seguidas de morte. Os profissionais, portanto, ficam sujeitos às sanções do código de ética e órgãos de classe ${ }^{12}$.

Desta forma, fica evidente que se deve promover a educação continuada destes profissionais, resgatando conhecimentos gerais sobre transmissão das doenças e as formas para evitar sua disseminação. 


\section{CONSIDERAÇÕES FINAIS}

Com o estudo, percebemos a renovação contínua do tema, tanto a nível nacional quanto internacional, visto que as infecções adquiridas em instituições de saúde estão entre as mais importantes causas de morte e aumento da morbidade dos doentes hospitalizados em todo o mundo, o que constitui um peso significativo tanto para os doentes como para a saúde pública $^{2,3,11}$.

Nota-se também a participação ativa das enfermeiras em programas de controle de infecção hospitalar e em pesquisas relacionadas ao assunto. Isto evidencia a conscientização desses profissionais na prevenção das infecções nosocomiais como responsabilidade nos cuidados de enfermagem no contexto dos cuidados na saúde e que só se alcançam resultados positivos com o trabalho em equipe. Isto inclui todos profissionais que prestam os cuidados diretos ao paciente, a gestão, as instalações, o aprovisionamento de materiais, equipamentos e produtos.

Um manual de prevenção da infecção nosocomial, com a compilação das instruções e práticas recomendadas para 0 cuidado dos pacientes, deve ser desenvolvido e atualizado pela equipe de controle de infecção, revisto e aprovado pela Comissão de Controle de Infecção Hospitalar (CClH).

Estes programas são eficazes desde que sejam abrangentes e incluam atividades de vigilância e prevenção, assim como a formação dos profissionais. Portanto, consideramos a graduação em enfermagem um momento de formação profissional propício para a introdução de conceitos de prevenção e controle de infecção hospitalar.

E também é essencial que haja um apoio eficaz em níveis regional e nacional aos hospitais e outras instituições de saúde. Deve ser desenvolvido plano de ação anual para a avaliação e promoção de cuidados de saúde de qualidade, isolamento apropriado, assim como esterilização e outras práticas, formação dos profissionais e vigilância epidemiológica. A administração deve disponibilizar os recursos suficientes para a concretização do plano, a fim de reduzir os custos econômicos decorrentes do prolongamento da hospitalização.

\section{Referências}

1.Ministério da Saúde (BR). Agência Nacional de Vigilância SanitáriaANVISA.: controle de infecções em serviços de saúde. Brasília (DF); 2001.

2.Ministério da Saúde (BR). Prevenção de infecções adquiridas no hospital: um guia prático. Insarj. [on-line] 2005 mar/abr; [citado 30 abril 2005]; [1 tela]. Disponível em: www.insarj.pt/site/resources/ images/PNCl/man oms.pdf

3.Ministério da Saúde (BR). Agência Nacional de Vigilância SanitáriaANVISA. Controle de Infecção. [on-line] 2005 jun/jul; [citado 22 jul 2005]; [aprox. 4 telas]. Disponível em: www.anvisa.gov.br/correlatos/ serv/infec.htm

4.Lakatos EM, Marconi MA. Metodologia do trabalho científico. $6^{\text {a }}$ ed. São Paulo (SP): Atlas; 2001.

5.Eco U. Como se faz uma tese. $21^{\text {a }}$ ed. São Paulo (SP): Perspectiva; 2007.
6. Hinrichsen SL. Biossegurança e controle de infecções: risco sanitário hospitalar. Rio de Janeiro (RJ): Medsi; 2004.

7.Melo DS, Souza ACS, Tipple AFV, Neves ZCP, Pereira MS. Compreensão sobre precauções padrão pelos enfermeiros de um hospital público de Goiânia. Rev Latino-am Enfermagem 2006 set/out; 14(5): 720 27.

8.Neves ZCP, Tipple AFV, Souza ACS, Pereira MS, Melo DS, Ferreira LR. Higienização das mãos: 0 impacto de estratégias de incentivo à adesão entre profissionais de saúde de uma unidade de terapia intensiva neonatal. Rev Latino-am Enfermagem 2006 jul/ago; 14(4): 546-52.

9.Cataneo C, Silveira CA, Simpionato E, Camargo FC, Queiroz FA, Cagnin MC. 0 preparo da equipe cirúrgica: aspecto relevante no controle da contaminação ambiental. Rev Latino-am Enfermagem 2004 mar/abr; 12(2): 283-86.

10.Tipple AFV, Pereira MS, Hayashida M, Moriya TM, Souza ACS. 0 ensino do controle de infecção: um ensaio teórico-prático. Rev Latinoam Enfermagem 2003 mar/abr; 11(2): 245-50.

11.Lacerda RA. Produção científica nacional sobre infecção hospitalar e a contribuição da enfermagem: ontem, hoje e perspectivas. Rev Latino-am Enfermagem 2002 jan/fev; 10(1): 55-63.

12.Bolick D, Brady C, Bruner DW, Edelstein S, Lane K, McLaughlin MB, et al. Segurança e controle de infecção. Rio de Janeiro (RJ): Reichmann \& Affonso; 2000. 
